\title{
Deep-water cycling and the Magmatic History of the Earth
}

\author{
Johnny Seales ${ }^{1}$, Adrian Lenardic ${ }^{1}$, and Mark Richards ${ }^{2}$ \\ ${ }^{1}$ Rice University \\ ${ }^{2}$ University of Washington
} 7 free to contact any of the authors.

\section{\& Abstract}

This is a preprint of the manuscript currently in review at Science Advances. Subsequent versions of this manuscript may contain different content. Should you have any questions or feedback, please feel

Earth is a magmatically active planet. Magmatism connects Earth's interior to its atmosphere, hydrosphere, and biosphere through cycling of volatiles, greenhouse gasses, and nutrients [18]. Earth's magmatic history is intertwined with its thermal and tectonic evolution. How magmatism has evolved and been maintained in the face of planetary cooling remains an open question. We address this question using data-constrained deep-water cycling and thermal history models. We track magmatic potential using a homologous temperature: the ratio of upper mantle to melting temperatures. After an initial decline, homologous temperature is buffered at a nearly constant value from roughly 2.5-2.0 Ga to the present day. Melt buffering reflects two factors: 1) The dependence of melting temperature on water content [21], and 2) The dependence of mantle viscosity on temperature and water content [15, 31, 27].

The latter allows solid Earth evolution to self-regulate via feedbacks that keep mantle viscosity at a near constant value. Self-regulation occurs even though the mantle remains far from thermal equilibrium, consistent with heat flow data. The added feedback from water-dependent melting allows magmatism to be co-buffered over geological time. This indicates that coupled thermal and water cycling feedbacks have maintained melting on Earth and associated volcanic/magmatic activity. Magmatic self-regulation affects not only the lifetime of geological activity on Earth but also, to the degree that planetary life connects to volcanic activity, the maintenance of conditions favorable for life. 
The cycling of water between Earth's surface and interior maintains volcanic activity and buffers longterm thermal and tectonic evolution.

\section{${ }_{28}$ Introduction}

It has long been noted that the temperature of Earth's shallow mantle is remarkably close to the melting temperature of rock [39]. That proximity (Figure 1a) is critical to Earth's current volcanic activity. It could be a coincidence, in which case our planet's magmatic/volcanic activity will decline as it continues to cool. More likely, it could reflect some form of feedback(s) that allow the Earth's cooling and magmatic potential to be co-buffered. Magmatic/volcanic regulation over geologic time has not generally been considered. However, data constraints on melt fraction from continental arcs indicate that it is a viable hypothesis (Figure 1b). The melting data from Brenhin Keller and Schoene [2] are consistent with the the idea that Earth experienced a decline in magmatic potential since early in its history, leveling off to quasi-steady state around 2.0 to 2.5 billion years ago. A quasi-steady state evolution in the face of continued planetary cooling requires some form of regulating feedback(s). This connects magmatic history to another long standing issue: Is Earth's evolution self-regulated?

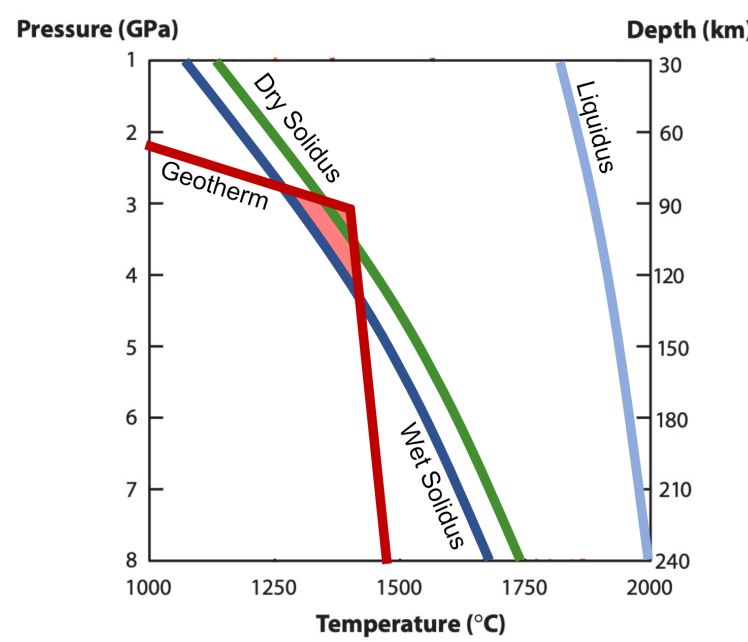

(a)

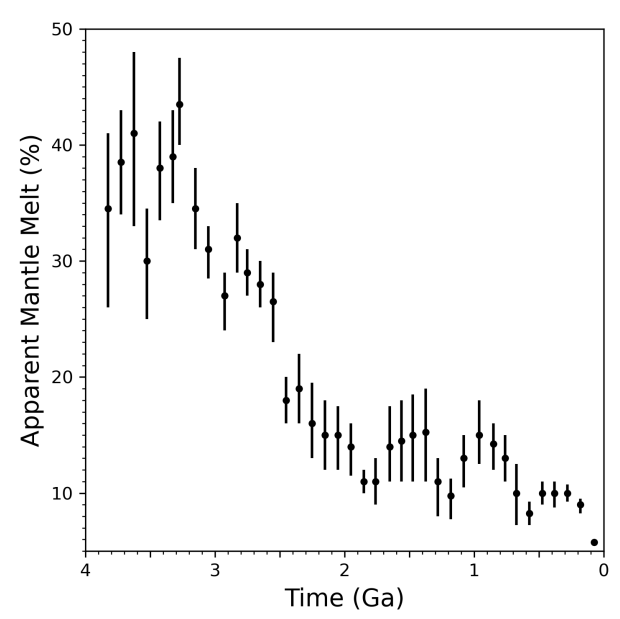

(b)

Figure 1: (a)The position of the Earths mean oceanic geotherm (red) relative to the dry (green) and wet solidus (light blue) and liquidus (dark blue) of the upper mantle. The geotherm is calculated following the procedure in the Methods and assuming a present day heat flow of $35 \mathrm{TW}$ and potential temperature of $1350{ }^{\circ} \mathrm{C}$. The melting curves represent the dry solidus [14] and wet solidus [21] assuming 2.5 OMs in the mantle - roughly the median value found in our analysis (Figure 2c). (b) Melt fraction from continental arcs over geologic history. [2, 22] 
A class of planetary cooling models do allow for thermal self-regulation [40, 8, 34]. A feedback between temperature and planetary cooling rate, facilitated by the temperature-dependence of mantle viscosity, allows the internal temperature of Earth to track the decay of radiogenic heat sources. This maintains the ratio of heat generation to heat loss, termed the Urey ratio $(U r)$, near unity. However, this regulation mechanism is not connected to magmatic evolution [39]. More problematic, such models cannot account for updated constraints on Earth's cooling history [4, 24]. In particular, data constraints place $U r$ between 0.2 and 0.5 [19], i.e., heat loss and heat generation appear to be far from equilibrium.

Self-regulation relates to the characteristic reactance time of the solid planet $[25,38]$. Reactance time characterizes system response to deviations from a secular trend. The secular trend is associated with the time scale over which the driving energy source for mantle convection changes due to radiogenic decay. Cooling histories that allow for thermal self-regulation have short reactance times relative to the decay time. Short thermal reactance times cannot lead to low $U r$ values, as they damp large deviations from thermal equilibrium. This, in turn, has been used to argue that mantle convection is not selfregulated, which has implications not only for understanding our own planet's evolution, but also for comparative planetology [25]. Although this argument is robust for thermal self-regulation, it does not rule out self-regulation altogether.

The ability of a planet to self-regulate depends on a relationship between the vigor of mantle convection, as characterized by the mantle Rayleigh number $(R a)$, and convective heat flux $(N u)$. That relationship is given by

$$
N u \sim R a^{\beta}
$$

where

$$
R a=\frac{\rho g \alpha \Delta T Z^{3}}{\kappa \eta}
$$

and $\rho$ is density, $\alpha$ is thermal expansivity, $g$ is the acceleration due to gravity, $\Delta T$ is the superadiabatic driving temperature, $Z$ is the thickness of the convecting layer, $\kappa$ is the thermal diffusivity and $\eta$ is the mantle viscosity. Models that allow for thermal self-regulation invoke a strong relationship between $N u$ and $R a$ [40]. That is, $\beta$ values near the high-end limit of $1 / 3$ [34]. Physically, this means that mantle viscosity is the dominant resistance to tectonic plate overturn. Conceptually, the regulating feedback works as follows: If fluctuations cause heat flux to become low relative to internal heat generation then the mantle will heat up, viscosity will decrease, and heat flux will increase (due to increased tectonic plate overturn associated with lower viscous resistance). The feedback operates on a short time scale relative to secular radiogenic heat source decay. As a result, interior cooling evolves along a series of quasi-equilibrium steps [8]. This is equivalent to $U r$ remaining near unity. Models with $\beta \leq 0$ can match $U r$ constraints as they have long reactance times that allow the Earth to remain far from thermal 
equilibrium $[4,24,25]$. Low or negative $\beta$ indicates that the dominant resistance to plate motion does not come from mantle viscosity, but instead from the strength of plates and/or plate margins. This connects self-regulation to the balance of plate tectonic forces. That balance is not agreed upon and it is critical to developing a dynamic theory of plate tectonics [6].

Although classic thermal histories focused on thermal-regulation, the critical assumption at their core is viscosity-regulation. That is, changes in viscosity dominate changes in the Earth's Rayleigh number and, over time scales shorter than secular decay times, viscosity, and by association the Rayleigh number, can be approximated as remaining constant. This is a critical assumption in using $N u \sim R a^{\beta}$ scaling relationships to begin with, as they are based on theory, experiments, and/or numerical simulations carried out under constant $R a$ values [32]. If viscosity depends only on temperature, then a lack of thermal-regulation rules out self-regulation. If that is not the case then self-regulation remains viable. The dependence of mantle viscosity on water opens this possibility [28, 27]. It also allows for potential co-regulation of mantle melting, as water content affects the melting temperature of rock [21].

The first generation of thermal history models that considered the role of water predicted $U r$ values greater than one [17] or comparable to classic models [29]. The former enforced a net loss of water from the Earth's interior. The latter assumed that $U r$ should be 0.8 and, as such, calibrated free parameters to keep mantle water content nearly constant. Crowley et al. [7] elegantly showed that a larger range of behavior is possible if the system allows for imbalances in mantle dewatering $(D)$ and rewatering $(R)$. Mantle dewatering occurs at mid-ocean ridges. The rate of mantle water loss depends on the relative positioning of the solidus and geotherm. Mantle rewatering occurs at subduction zones, where descending slabs carry some of their bound water into the mantle. How much water the slab can carry scales with its thickness, which will increase as the mantle cools. If mantle viscosity depends on temperature $(T)$ and mantle water content $(\chi)$, then the time rate of change of mantle viscosity can be written as

$$
\frac{d \eta}{d t}=\frac{\partial \eta}{\partial T} \frac{d T}{d t}+\frac{\partial \eta}{\partial \chi} \frac{d \chi}{d t}
$$

94 Conservation of energy leads to

$$
\frac{d T}{d t}=\frac{1}{\rho C_{p} V}\left(H-Q_{s}\right)
$$

${ }_{95}$ where $C_{p}$ is specific heat, $V$ is mantle volume, $H$ is mantle heat production, and $Q_{s}$ is surface heat flow.

96 Conservation of mantle water content leads to

$$
\frac{d \chi}{d t}=\frac{1}{\rho V}(R-D)
$$

97 Following the assumption that viscosity remains statistically steady, relative to the time scale over which 
significant changes occur in internal heat generation, leads to an estimate for the Urey ratio given by

$$
U r \approx 1-\frac{\eta_{\chi}}{\eta_{T}} \frac{C_{p}}{Q_{s}}(R-D)
$$

where $\eta_{\chi}=\frac{\partial \eta}{\partial \chi}$ and $\eta_{T}=\frac{\partial \eta}{\partial T}$. If $R$ exceeds $D$, then the Earth can be out of thermal equilibrium and low values of $U r$ are viable without requiring a weak, or negative, relationship between surface heat loss and $R a$. The analysis of Crowley et al. [7] is significant in motivating our work, as it re-opens the possibility of planetary self-regulation. It did not, however, show that the Earth followed a self-regulated path, nor did it address magmatic evolution. In what follows we will do so using data-constrained thermal evolution models that allow for water cycling.

\section{Results}

Thermal history models have multiple free parameters and initial conditions. This can require millions of model paths to map parameter space, with the vast majority of paths falling outside of observational constraints $[38,36]$. The problem can be bridled via data assimilation. Here we develop and employ a novel data assimilation method applied to coupled thermal history and deep-water cycling models. The method directly builds in data constrained thermal history trajectories over geologic time (a full overview can be found in the Methods). The trajectories are constrained to match, within uncertainty, petrological data $[13,5,11]$. Figure 2a shows the subset of trajectories $(>250)$ that met a goodness of fit criteria (see Methods). The method also assimilates constraints on the present day Urey ratio. We varied the $U r$ between 0.2 and 0.5 [19]. Variable $\beta$ values are allowed for to test models with different resisting forces to tectonic plate motions. For each thermal trajectory we randomly sampled one-hundred different combinations of $U r$ and $\beta$ within the assigned bounds and inverted for mantle water content. This involved converting a forward model of coupled thermal and water history [37] into an inverse model. Present day surface water content was constrained to be one ocean mass equivalent (OM) and present day mantle viscosity was required to fall between $10^{19}$ and $10^{22} \mathrm{~Pa}$. With these constraints, the evolution of mantle and surface water content was determined throughout Earth's history. This procedure produced 10,000 mantle water evolution paths.

Figure $2 \mathrm{~b}$ shows the relative density of successful $U r-\beta$ space. Successful models preferentially gathered towards the lower $U r$ bound of Jaupart et al. [19]. Successful trajectories also required approximately $\beta \geq 0.2$. Figure 2c shows mantle water evolution. Model and data uncertainties demand that outputs be calculated, and plotted, as probability distributions, versus a single preferred path. The median of the distribution is depicted as a thick, black line. The darker region encompassing the median 


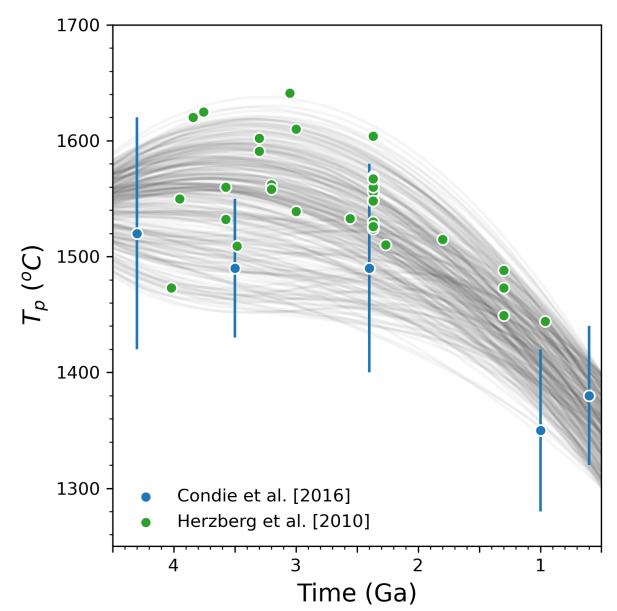

(a)

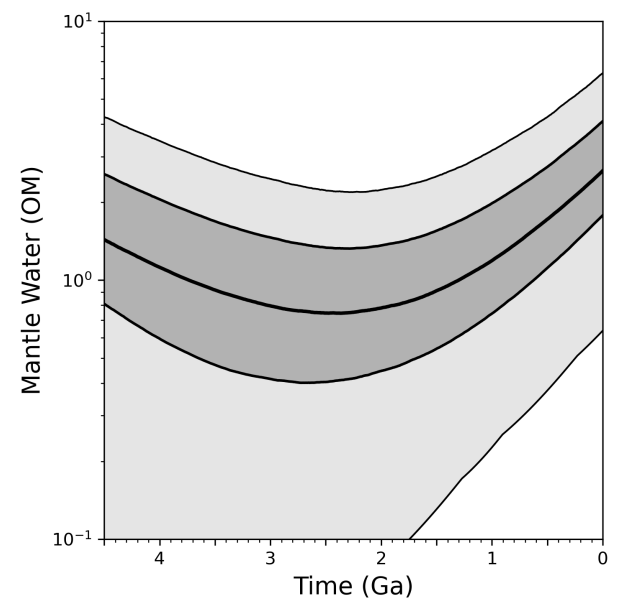

(c)

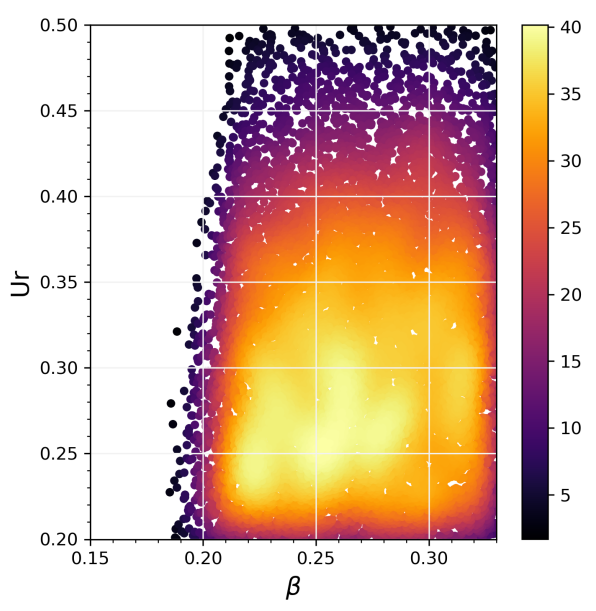

(b)

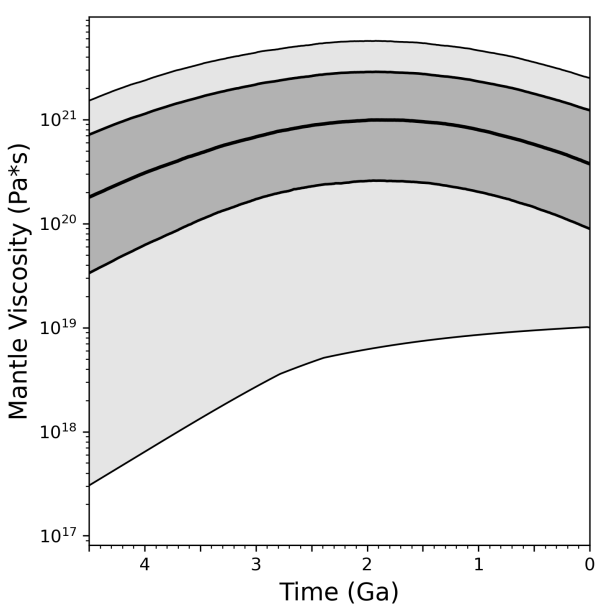

(d)

Figure 2: Thermal trajectories consistent with Earth data (a) used for obtaining inversion results (b-d). (b) successful $U r-\beta$ parameter space colored by relative point density with higher values meaning the density of points is larger. (c) Distribution of mantle water content and (d) mantle viscosity shown as distributions about their median value. The dark gray highlights values falling between the upper and lower quartiles and the lighter gray constraining the maximal and minimal limits.

is bounded by the upper and lower quartiles. The lighter regions extend from these quartiles to one and half times the interquartile range. The distribution shows that successful models experienced an early period of net mantle dewatering followed by net rewatering. The change occurred between two and three billion years ago. The timing aligns with the findings of Parai and Mukhopadhyay [33] and Seales and Lenardic [37]. Dong et al. [9] suggested a net rewatering over Earth's history by estimating the mantle water capacitance. This, however, defines an upper limit within some uncertainty. We know of no physical reasoning demanding that the mantle remain at this limit, and the majority of our results fall within or below their uncertainties. Figure 2d shows the evolution of mantle viscosity from successful models. In the absence of water, an expectation would be a monotonically increasing viscosity due to mantle cooling. However, successful models show an increase in mantle viscosity for roughly the first half 
of Earth's history followed by a milder decline. The rollover coincides with the change from net mantle dewatering to rewatering. Physically, this corresponds to a switch from hot and dry subduction to cold and wet subduction that cycles larger volumes of water into the mantle.

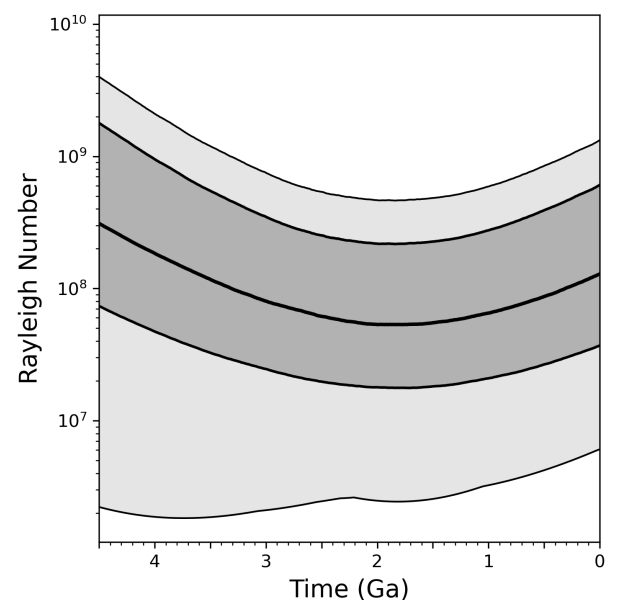

(a)

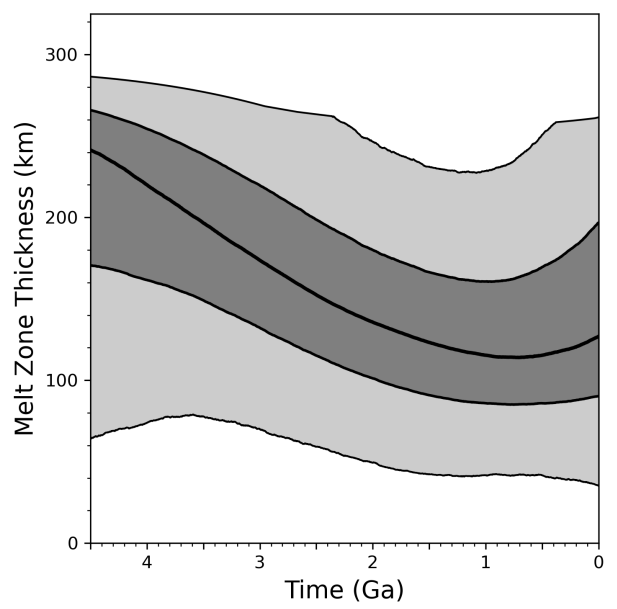

(c)

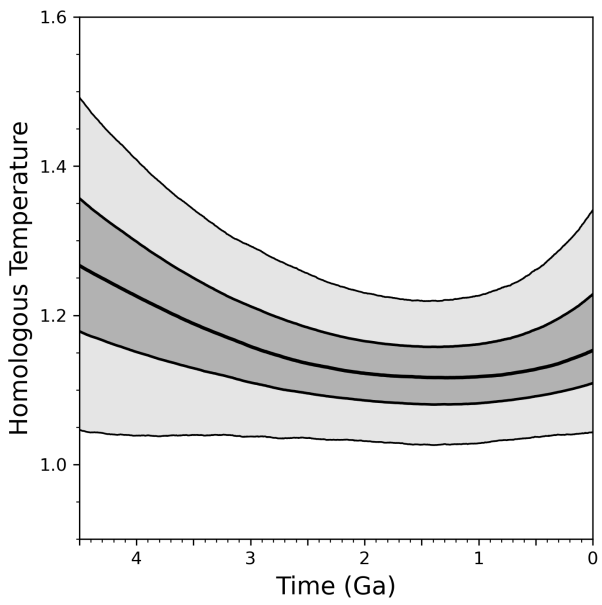

(b)

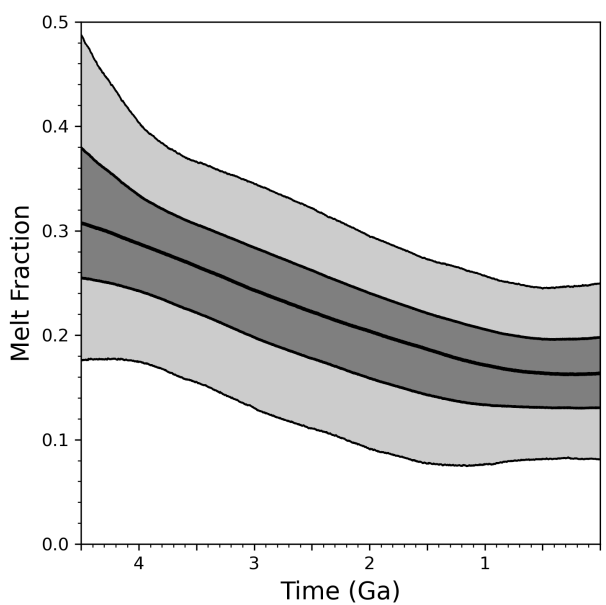

(d)

Figure 3: Measure of mantle self-regulation and co-regulation of mantle melting. (a) Inversion results showing $R a$ falling within a relatively narrow range throughout Earth's history. (b) Inversion results showing recent regulation of Earth's Homologous temperature. (c) Inversion results showing melt zone thickness. (d) Inversion results for melt fraction showing a decay following by a level off near present day. Each of the figures show results as distributions about their median value. The darker color highlights values falling between the upper and lower quartiles and the lighter color constraining the maximal and minimal limits.

The mild change of viscosity shown in Figure $2 \mathrm{~d}$ leads to a similar trend for the mantle Rayleigh number, a measure of convective vigor. Figure 3a plots model $R a$ evolution. The mild change in $R a$, in the face of a significant decline of internal radiogenic heating, is indicative of a self-regulated mantle evolution. In the absence of deep-water cycling, mantle cooling would lead to a a decrease in $R a$. A flat, and potentially increasing, $R a$ trend is consistent with observationally based inferences from passive margins that plate speeds have not been decreasing over geologic time and could, within data 
uncertainty, even be increasing [1]. It is also consistent with the conjecture, based on observational constraints on water transport beneath Japan arcs, that deep-water cycling could stabilize and prolong mantle convection and the associated geological activity of the Earth [16].

Another measure of self-regulation, beyond $R a$, is the homologous temperature $\left(T_{H}\right)$. We define $T_{H}$ as the ratio of two depth-dependent profiles: the mantle geotherm and the wet solidus. Figure 1a shows these profiles in black and blue, respectively. As the two profiles change with depth, we define $T_{H}$ at the maximum distance between the two curves (see Methods). In Figure 1a this point occurs at the change in slope of the geotherm, which is the base of the thermal lithosphere. The greater the thermal distance between the solidus and geotherm, the greater the value of $T_{H}$. When $T_{H}$ drops below unity, melting ceases.

Figure $3 \mathrm{~b}$ shows how $T_{H}$ evolves for successful models. The decrease over the first few billion years coincides with net mantle dewatering (Figure 2c). Decreasing $T_{H}$ is associated with melt zone thinning (Figure 3c). The change from net mantle dewatering to rewatering changes the behavior of $T_{H}$. The flattening of the slope around $2 \mathrm{Ga}$ in Figure $3 \mathrm{~b}$ indicates that the thermal distance between the solidus and geotherm remains nearly constant. Melt zone thickness also remains constant within $\pm 10 \mathrm{~km}$ (Figure 3c). This indicates that melt fraction can remain relatively constant over the same time period. Figure 3d shows that we indeed find a higher melt fraction early in Earth's history followed by a self-regulated period towards present. The onset of self-regulation is consistent with geochemical constraints on a switch from net mantle dewatering to rewatering [33]. It has been argued that this timing is also coincident with a change from dominantly mafic to felsic continental composition, which led to a rise of atmospheric oxygen $[26,10]$. This, in turn, is consistent with the onset of cold and wet subduction, which leads to melt regulation, also driving a switch toward the formation of felsic volcanism.

One might assume that an initial drop in $T_{H}$ over the first 2 billion years of evolution would be due to rapid initial mantle cooling $[2,22]$. However data constraints show that over this time, mantle cooling is mild, if at all, and accelerates subsequently (Figure 2a). This may run counter to intuition, but it is critical to Earth's present-day Urey ratio. A low $U r$ indicates that, at present, mantle heat flow is high relative to internal heat generation (i.e., the mantle is far from thermal equilibrium). This requires a period of relatively low mantle heat flow in the Earth's past to retain heat such that it is then available to supply elevated present day heat flow. Our successful models allow for this, while at the same time maintaining a strong relationship between $R a$ and convective heat flux via a switch from mantle dewatering, and associated mild cooling, to net mantle rewatering and an associated increased cooling rate. Over the accelerated cooling phase, thermal and water cycling feedbacks lead to a self-regulation of mantle melt potential as expressed by $T_{H}$ (Figure $3 \mathrm{~b}$ ). 


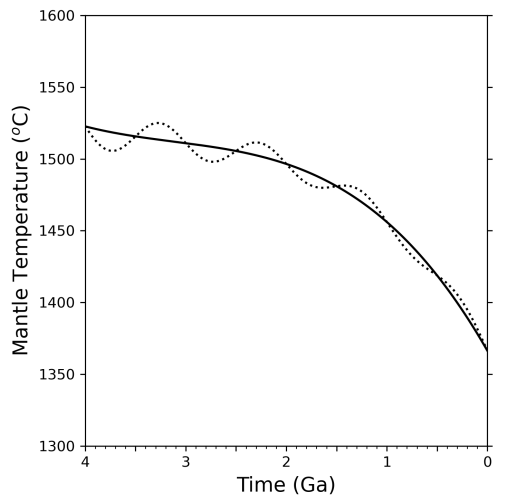

(a)

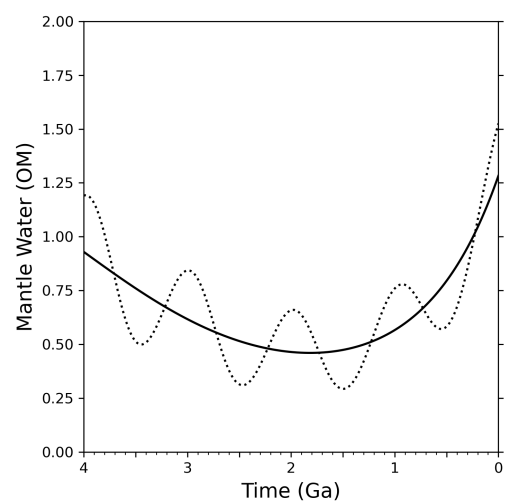

(b)

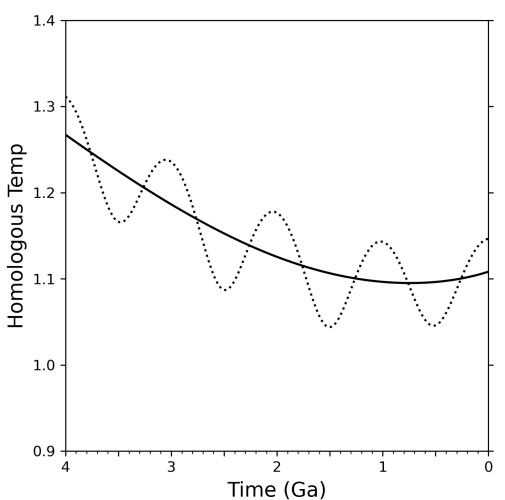

(c)

Figure 4: An example of how fluctuations within Earth's mantle convection and plate tectonic system can affect the mantle potential temperature (a), water content (b) and homologous temperature (c).

The trends of Figure 3 cannot extend indefinitely as interior cooling will eventually lead to $T_{H}$ dropping below unity. Exactly when this occurs depends on the future path of mantle temperature and water content. Given that the solidus depends on water content, the self-regulation mechanisms mapped by our models can delay melt shutdown relative to a dry planet or a planet that does not allow for two way water cycling. In principal, we could extend our models forward in time. The results would, however, be deceptive as we would be taking a data assimilation method outside of data constraints. This leads to increasing uncertainty the further a projection is taken outside of the data [23]. The constraints our models give on current conditions (e.g., mantle temperature, water content, $U r, \beta$ ) could be used as initial conditions and parameter constraints on forward models that are subject to a full uncertainty quantification $[38,36]$ to provide probability densities for the timing of melt shut down. That type of analysis would also need to consider the potential of cooling induced shifts in tectonic modes from plate tectonics to a single plate planet. That goes beyond the intent and scope of this paper (i.e., to investigate the hypothesis that mantle melting was self-regulated over the Earth's evolution to the present day).

Results thus far have shown the evolution of mean trends, together with uncertainties. Self-regulation does not, however, require that planetary variables remain on slowly varying mean paths. Fluctuations can occur but negative feedbacks tend to bring the system back toward a mean trend. The Earth's thermal-tectonic system allows for fluctuations due to, for example, the chaotic nature of mantle convection, changes in plate-dimensions, and the amalgamation/dispersal of super-continents. These fluctuations can lead to variations in deep-water cycling [20]. Fluctuations could also occur as a result of variations in the time scale of mixing water into the mantle [3]. Our methodology can deal with these possibilities. An example can demonstrate the effects of including fluctuations into our analysis by comparing smooth to fluctuating thermal paths (Figure 4a). The particular form of fluctuations is an example only. Figures $4 \mathrm{~b}$ and $4 \mathrm{c}$ show how thermal fluctuations effect water cycling and melt potential, respectively. The system maintains a self-regulated evolution but it does so in a statistical sense. This 
is consistent with the data of Keller and Schoene [22], which show fluctuations in melt fraction about a slowly varying mean trend over the last 2 billion years (Figure 1b). In principal, one could build in direct data constraints on, for example, fluctuations in mantle potential temperature [41] and/or mean plate subduction age [20]. That goes beyond our scope of demonstrating that self-regulation is robust in the face of thermal-tectonic fluctuations.

\section{Discussion}

Data-constrained models of Earth's thermal history indicate that coupled deep-water and thermal cycles can lead to a self-regulated mode of mantle convection with an associated self-regulation of the Earth's magmatic potential. In the more than five decades since the advent of a mobilist, plate-tectonic view of Earth dynamics, the notion that the solid-Earth's strong dependence of viscosity upon temperature should buffer variations in mantle internal temperature against the decay of secular radiogenic heat sources has dominated much work on thermal evolution of the mantle. Recently, that possibility has been questioned based on the recognition that the low value of the present-day Urey ratio is inconsistent with thermal self-regulation. However, when we incorporate the effects of mantle volatile concentration on melting, and hence viscosity, we find a broad and plausible range of models that are compatible with a more general form of self-regulation. Models that operate in that mode of self-regulation are compatible with constraints on the internal temperature, volatile content, viscosity, and magmatic history of the Earth, as well as with low values for Ur. They also imply that the dominant resistance to the motion of tectonic plates comes from mantle viscosity. Within uncertainty, the strength of plates and/or plate margins can play a non-trivial, if not dominant, role (Figure 2b). Critically, successful models imply a balance of resisting forces that is consistent with more detailed models of subduction zone dynamics [12].

Fundamentally, partial melting in Earth's upper mantle (asthenosphere), due to the presence of volatiles, mode locks the mantle toward higher (nearer wet solidus) interior temperatures, and hence higher heat flow and lower Ur, relative to a planet that might otherwise have interior temperatures that more closely track the secular decrease in internal heat sources. This conclusion is likely to have important implications for Earth's plate tectonic style of mantle convection, in that partial melting in the asthenosphere profoundly influences the existence and behavior of plates and plate motions - indeed sound arguments can be made that the long-term persistence of plate tectonics on Earth requires the persistence of a partially-molten uppermost mantle, which in turn is necessary in order for the melt-related selfregulation mechanism we have explored to be effective. We further suggest that exploration of both constraints for and models of mantle dewatering and rewatering (related primarily to volatile processing at ridges and subduction zones) should shed further light on mantle evolution and self-regulation. 


\section{Materials and Methods}

\section{Assimilating data into parameterized thermal history models}

The average mantle temperature changes with time $\left(\dot{T}_{m}\right)$ according to the balance of heat produced within $(H)$ and lost from $(Q)$ the mantle:

$$
\rho V c_{p} \dot{T}_{m}=H-Q
$$

where $V$ and $c_{p}$ are the volume and heat capacity of the mantle, respectively [35]. The amount of heat produced by the decay of radiogenic elements within the mantle scales as

$$
H=V h_{i} \exp (-\lambda t)
$$

where $h_{i}$ is the initial heat generation rate, $\lambda$ is the decay constant, and $t$ is time. The total amount of heat lost by convective cooling is

$$
Q=A q_{m}
$$

where $A$ is the surface area of the convecting mantle and $q_{m}$ the convective heat flux. Non-dimensional heat flux $(N u)$ scales with the Rayleigh number $(R a)$, a measure of convective vigor, according to

$$
N u=\frac{q_{m}}{q_{\text {cond }}}=\left(\frac{R a}{R a_{c}}\right)^{\beta}
$$

where $q_{c o n d}$ is the amount of heat lost were it transferred solely by conduction through the entire layer, $R a_{c}$ is the critical Rayleigh number and $\beta$ is a scaling exponent. The parameter $\beta$ varies between models that make different assumptions as to the dominant forces resisting tectonic plate motion (see Seales and Lenardic [36] for a fuller discussion of what different $\beta$ values mean for mantle convection). Fourier's law states that

$$
q_{\text {cond }}=k \frac{\Delta T}{Z}
$$

where $k$ is the thermal conductivity of the mantle, $Z$ is the depth of the convecting layer, and $\Delta T$ is the temperature difference driving convection. The latter is the difference between the surface temperature $\left(T_{s}\right)$ and the average mantle temperature. Combining Equations 10 and 11 and rearranging, the convective heat flux is

$$
q_{m}=k \frac{\Delta T}{Z}\left(\frac{R a}{R a_{c}}\right)^{\beta}
$$


where $R a$ is

$$
R a=\frac{\rho g \alpha \Delta T Z^{3}}{\kappa \eta}
$$

and $\alpha$ is the mantle thermal expansivity, $\kappa$ is the mantle thermal diffusivity and $\eta$ is the mantle viscosity.

Viscosity depends on temperature and water content according to

$$
\eta=\eta_{o} A_{c r e}^{-1}\left[\exp \left(c_{0}+c_{1} \ln \chi_{m}+c_{2} \ln ^{2} \chi_{m}+c_{3} \ln ^{3} \chi_{m}\right)\right]^{-r} \exp \left(\frac{E}{R T_{m}}\right)
$$

where $\eta_{o}$ is a scaling constant, $c_{1}, c_{2}$ and $c_{3}$ are empirically determined constants [27], $r$ is the water fugacity exponent, $A_{c r e}$ is a material constant, $E$ is the activation energy for creep and $R$ is the universal gas constant. In Equation 14, $\chi_{m}$ has units $H / 10^{6} \mathrm{SI}$.

Combining Equations 7 to 13, we find that the change in mantle temperature evolves according to

$$
\dot{T}_{m}=\frac{1}{\rho c_{p}}\left[\sum_{i=1}^{n} h_{i} \exp \left(-\lambda_{i} t\right)-\frac{A}{V} \frac{k \Delta T}{D}\left(\frac{\rho g \alpha \Delta T D^{3}}{R a_{c} \kappa \eta}\right)^{\beta}\right]
$$

Rearranging to isolate mantle viscosity, Equation 15 becomes

$$
\eta=\frac{R a_{c} \kappa}{\rho g \alpha \Delta T D^{3}}\left[\frac{V}{A} \frac{D}{k \Delta T}\left(\sum_{i=1}^{n} h_{i} \exp \left(-\lambda_{i} t\right)-\rho c_{p} \dot{T}_{m}\right)\right]^{-\frac{1}{\beta}}
$$

Equations 14 and 16 have $\eta$ isolated. As such, we can use these equations to estimate $\chi_{m}$. We use Herzberg et al. [13] and Condie et al. [5] as constraints on $T_{m}$. Viable thermal paths based on those $T_{m}$ constraints (Section) provide constraints on the time derivative of mantle temperature $\left(\dot{T}_{m}\right)$. The Urey ratio, defined as $U r=H / Q$, serves as a data constraint. Given a present day estimate of $Q$, we can calculate present day $H$. Substituting this value of $H$ into Equation 8, we can rearrange and solve for $h_{i}$, which will determine the rate of radiogenic heating for that model. Given the parameters in Table 1 and the constraints laid out here, $\chi_{m}$ remains the only unknown in Equations 14 and 16. We initially estimate the mantle water content and then iteratively adjust this value until Equations 14 and 16 are within some tolerance $(\epsilon)$ of each other. We verified the inversion results against the outputs of forward models. The global maximum inversion error remained less than one percent and the average inversion error remained below 0.01 percent over the modelled time domain. 


\section{Constructing Thermal Trajectories}

The method detailed above requires a thermal trajectory as input. One can imagine many trajectories satisfying the uncertainties in estimated mantle potential temperature (Figure 2a). As such, we constructed a number of data constrained thermal trajectories, each of which passed through strategic control points $\left(P_{i}\right)$ defined by the coordinates $\left(t_{i}, T_{i}\right)$, with time $t_{i}$ in billions of years before present and mantle potential temperature $T_{i}$ in ${ }^{\circ} C$. The control points $P_{o}$ and $P_{f}$ define the initial and present day temperatures, respectively. We required each thermal trajectory pass through at least one intermediate control point $P_{m 1}$, which coincides with the rollover in the Herzberg et al. [13] and the change in slope of Condie et al. [5]. Table 1 lists the uncertainties we considered for each control point. We drew random samples from uniform distributions defined by these bounds. These samples served as the starting point of our thermal trajectory. If the sampling resulted in $T_{m 1}>T_{o}$, we defined the thermal trajectory using the quadratic

$$
T(t)=\alpha_{1} t^{2}+\alpha_{2} t+\alpha_{3}
$$

We determined the constants $\alpha_{i}$ by using the control points $P_{o}, P_{m 1}$ and $P_{f}$ to form a system of three equations with three unknowns. If sampling resulted in $T_{m 1}<T_{o}$, we defined the thermal trajectory using the cubic

$$
T(t)=\alpha_{1} t^{3}+\alpha_{2} t^{2}+\alpha_{3} t+\alpha_{4}
$$

Solving for $\alpha_{i}$ required a system of equations based on four control points. To account for this, we introduced $P_{m 2}$ such that $t_{m 2}=t_{m 1}-\tau_{m t}$ and $T_{m 2}=T_{m 2}-\tau_{m T}$. Here $\tau_{m t}$ and $\tau_{m T}$ represent offset times and temperatures. These allow for flattening of the thermal trajectory after an initial temperature decline, which can occur when water and thermal cycles effect mantle viscosity [37].

We required that each thermal trajectory fit the data of Herzberg et al. [13] and Condie et al. [5] within some measure of goodness. We used a reduced chi-squared statistic, the chi-square $\left(\chi^{2}\right)$ per degree of freedom $(\nu)$. We adopt $\chi^{2}$ as traditionally defined:

$$
\chi^{2}=\sum_{t} \frac{[D(t)-M(t)]^{2}}{\sigma(t)} .
$$

This cumulatively measures the error $(\sigma(t))$ normalized difference between the data $(D(t))$ and modeled thermal trajectory $(M(t))$. For measuring the goodness of fit we included all data points from both data sets. This gave us a total of 38 data points $\left(N_{d}\right)$. The definition for degrees of freedom is: $\nu=N_{d}-N_{\alpha}+N_{P}$ given the number of parameters $\left(N_{\alpha}\right)$ and control points $\left(N_{P}\right)$. We only kept thermal trajectories that had $\chi^{2} / \nu<=1$. Using this method, we found a median accepted value of 0.98 . As this is nearly unity, so the thermal paths approximate the data error variance without over-fitting. 
To mimic a fluctuating mantle temperature, we constructed fluctuations $\left(T_{f}\right)$ that followed the form of an exponentially damped sine wave, which is of the form

$$
T_{f}(t)=A_{f} e^{-\lambda_{f} t} \sin \left(\frac{2 \pi}{P_{f}} t\right)
$$

where $A_{f}$ is the amplitude of the sine wave, $\lambda_{f}$ is the decay constant, $P_{f}$ is the period and $t$ is time, in billion of years. We set $A_{f}$ to one percent of the initial mantle potential temperature, $\lambda_{f}$ to 0.22 Gyr ${ }^{-1}$ and $P_{f}$ to 1 Gyr. We then superimposed $T_{f}$ on top of a path defined as above. We still enforced the condition that $\chi^{2} / \nu<=1$. We do no pretend to know what path fluctuations follow. They likely follow something much more complicated than presented here. However, we believe that the qualitative form of our findings will hold. An exact description of the fluctuations is beyond the scope of this paper. How to account for them in forward modeling was covered by Seales et al. [38]. Regardless, choosing any other path would find the same qualitative conclusions presented herein.

\section{Homologous Temperature}

Our analysis relied on the geotherm consisting of two elements: a shallow conductive profile through the mantle lithosphere and a convective profile beneath it. For a given value of $T_{p}$ and $\chi_{\mathrm{H}_{2} O}$, we can calculate $q_{m}$ according to Equation 12. We can rearrange Fourier's Law to give the conductive profile according to

$$
T_{\text {cond }}(z)=\frac{q_{m}}{k_{m}} z+T_{s}
$$

The convective profile is the mantle adiabat. We used a linearized version of Mckenzie and Bickle [30] above to convert from $T_{m}$ to $T_{p}$. We can also use this adiabat to construct the convective element of the geotherm according to

$$
T_{\text {conv }}(z)=T_{m}\left[1-\frac{g \alpha}{c_{p}}\left(\frac{R_{p}-R_{c}}{2}-z\right)\right]
$$

The conductive and convective profiles intersect at the base of the lithosphere $\left(H_{L}\right)$ :

$$
H_{L}=\left[T_{m}-T_{s}-\frac{T_{m} g \alpha}{2 c_{p}}\left(R_{p}-R_{c}\right)\right]\left(\frac{q_{m}}{k_{m}}-\frac{T_{m} g \alpha}{c_{p}}\right)^{-1}
$$

20 In our analysis we compared the geotherm to the solidus. We used the dry solidus of Hirschmann [14]. We accounted for water suppressing the dry solidus using the parameterization of Katz et al. [21]:

$$
T_{\text {sol }}(z)=A_{1}+A_{2} * \rho g z+A_{3}(\rho g z)^{2}-\Delta T+273
$$




$$
\Delta T\left(\chi_{\mathrm{H}_{2} \mathrm{O}}\right)=K \chi_{\mathrm{H}_{2} \mathrm{O}}^{\gamma}
$$

323

Melt zone thickness $\left(H_{M}\right)$ is defined as the vertical difference between the two points where the geotherm and solidus intersect. The shallower point defines the top of the melt zone $\left(H_{T}\right)$. Equating Equations 21 and 21 and gathering like therms gives the quadratic

$$
\begin{aligned}
A_{T} z^{2} & +B_{T} z+C_{T}=0 \\
A_{T} & =A_{3}(\rho g)^{2} \\
B_{T} & =A_{2} \rho g-\frac{q_{m}}{k_{m}} \\
C_{T} & =A_{1}-K_{H_{2} O}^{\gamma}+273-T_{s}
\end{aligned}
$$

331

This quadratic has two roots, one above the surface (unphysical) and one at depth. The one at depth defines $\left(H_{T}\right)$ and is given by

$$
H_{T}=\frac{-B_{T}-\sqrt{B_{T}^{2}-4 A_{T} C_{T}}}{2 A_{T}}
$$


We found the base of the melt zone $\left(H_{B}\right)$ by equating the convective profile (Equation 21) with the solidus (Equation 24). Grouping like terms and gathering gives the quadratic

$$
\begin{gathered}
A_{B} z^{2}+B_{B} z+C_{B}=0 \\
A_{B}=A_{3}(\rho g)^{2} \\
B_{B}=A_{2} \rho g-\frac{g \alpha}{c_{p}} T_{m} \\
C_{B}=A_{1}-K_{H_{2} O}^{\gamma}+273-T_{m}\left[1-\frac{g \alpha}{c_{p}}\left(\frac{R_{p}-R_{c}}{2}\right)\right]
\end{gathered}
$$
such, we calculate the melt fraction $(\phi)$ at each depth according to

$$
\phi=\frac{T(z)-T_{\text {sol }}(z)}{T_{l i q}(z)-T_{\text {sol }}(z)}
$$

$$
H_{B}=\frac{-B_{B}+\sqrt{B_{B}^{2}-4 A_{B} C_{B}}}{2 A_{B}}
$$

Data constrain the solidus to a depth of $300 \mathrm{~km}$. We set this as a hard maximum limit for $H_{B}$.

\section{Calculating Melt Fraction}

The distance between the the solidus and geotherm determines the amount of melt produced. The distance between the solidus and geotherm varies between the top and bottom of the melt zone. As

assuming that the melt fraction increases linearly between the solidus and liquidus $\left(T_{l i q}\right)$. We integrate Equation 37 over the entire melt zone and normalize by melt zone thickness to obtain an estimate of average melt fraction $(\bar{\phi})$. 
Table 1: Model parameters and values

\begin{tabular}{|c|c|c|c|}
\hline Parameter & Description & Value & Unit \\
\hline$\rho$ & Mantle density & 3000 & $\mathrm{~kg} / \mathrm{m}^{3}$ \\
\hline$c_{p}$ & Mantle heat capacity & 1400 & $\mathrm{~J} /(\mathrm{kg} \mathrm{K})$ \\
\hline $\mathrm{k}$ & Mantle thermal conductivity & 4.2 & $\mathrm{~W} /(\mathrm{m} \mathrm{K})$ \\
\hline$\alpha$ & Mantle thermal expansivity & $3 \times 10^{-5}$ & $\mathrm{~K}^{-1}$ \\
\hline$\kappa$ & Mantle thermal diffusivity & $10^{-6}$ & $\mathrm{~m}^{2} / \mathrm{s}$ \\
\hline$\lambda$ & Radiogenic decay constant & $3.4 \times 10^{-10}$ & $\mathrm{yr}^{-1}$ \\
\hline$Q_{i}$ & Present day mantle heat flow & $35 x 10^{12}$ & $\mathrm{~W}$ \\
\hline $\mathrm{Ur}$ & Present day Urey ratio & $0.2-0.5$ & - \\
\hline$\beta$ & Convective scaling exponent & $0.15-0.33$ & - \\
\hline$R a_{c}$ & Critical Rayleigh number & 1100 & - \\
\hline$T_{s}$ & Surface Temperature & 300 & $\mathrm{~K}$ \\
\hline $\mathrm{g}$ & Acceleration due to gravity & 9.8 & $\mathrm{~m} / \mathrm{s}^{2}$ \\
\hline$R_{p}$ & Radius of Earth's surface & 6371000 & $\mathrm{~m}$ \\
\hline$R_{c}$ & Radius of Earth's core & 3471000 & $\mathrm{~m}$ \\
\hline $\mathrm{Z}$ & Thickness of convecting layer & 2900000 & $\mathrm{~m}$ \\
\hline$\eta_{o}$ & Viscosity constant & $1.7 x 10^{17}$ & Pa s \\
\hline$A_{\text {cre }}$ & Material constant & 90 & $\mathrm{MPa}^{-r / s}$ \\
\hline$C_{0}$ & Empirically determined viscosity constant & -7.98 & - \\
\hline$C_{1}$ & Empirically determined viscosity constant & 4.35 & - \\
\hline$C_{2}$ & Empirically determined viscosity constant & -0.57 & - \\
\hline$C_{3}$ & Empirically determined viscosity constant & 0.03 & - \\
\hline $\mathrm{E}$ & Creep activation energy & $4.8 \times 10^{5}$ & $\mathrm{~J} / \mathrm{mol}$ \\
\hline $\mathrm{R}$ & Universal gas constant & 8.314 & $\mathrm{~J} / \mathrm{mol}$ \\
\hline $\mathrm{r}$ & Water fugacity exponent & 1.2 & - \\
\hline$T_{o}$ & Starting mantle temperature & $1400-1800$ & ${ }^{\circ} \mathrm{C}$ \\
\hline$T_{f}$ & Present day mantle temperature & $1300-1400$ & ${ }^{\circ} \mathrm{C}$ \\
\hline$T_{m 1}$ & Rollover temperature & $1450-1650$ & ${ }^{\circ} \mathrm{C}$ \\
\hline$\tau_{m T}$ & Rollover temperature & $-5-25$ & ${ }^{\circ} \mathrm{C}$ \\
\hline$t_{o}$ & Initial model time & 0 & Gyr \\
\hline$t_{f}$ & Final model time & 4.5 & Gyr \\
\hline$t_{m 1}$ & Intermediate model time & $1.25-2.5$ & Gyr \\
\hline$\tau_{m t}$ & Intermediate model time & $0.75-0.25$ & Gyr \\
\hline$A_{f}$ & Temperature fluctuation amplitude & $1 \%$ & ${ }^{\circ} \mathrm{C}$ \\
\hline$\lambda_{f}$ & Temperature fluctuation decay constant & $1 / 4.5$ & $\mathrm{Gyr}^{-1}$ \\
\hline$P_{f}$ & Temperature fluctuation frequency & 1 & $\mathrm{Gyr}^{-1}$ \\
\hline$A_{1}$ & Anhydrous solidus calibration constant & 1085.7 & ${ }^{o} \mathrm{C}$ \\
\hline$A_{2}$ & Anhydrous solidus calibration constant & 132.9 & ${ }^{o} \mathrm{C} \mathrm{GPa}^{-1}$ \\
\hline$A_{3}$ & Anhydrous solidus calibration constant & -5.1 & ${ }^{\circ} \mathrm{C} \mathrm{GPa}^{-2}$ \\
\hline $\mathrm{K}$ & Hydrous solidus calibration constant & 43 & ${ }^{\circ} \mathrm{C} \mathrm{wt} \%^{-\gamma}$ \\
\hline$\gamma$ & Hydrous solidus scaling exponent & 0.75 & - \\
\hline$\epsilon$ & $\chi$ convergence tolerance & $10^{-8}$ & $H / 10^{6} \mathrm{Si}$ \\
\hline $\mathrm{OM}$ & Present day ocean mass equivalent & $1.39 \times 10^{2} 1$ & $\mathrm{~kg}$ \\
\hline
\end{tabular}




\section{References}

[1] D. C. Bradley. Passive margins through earth history. Earth-Science Reviews, 91(1-4):1-26, 2008. ISSN 00128252. doi: 10.1016/j.earscirev.2008.08.001. URL http://dx.doi.org/10.1016/j . earscirev.2008.08.001.

[2] C. Brenhin Keller and B. Schoene. Statistical geochemistry reveals disruption in secular lithospheric evolution about 2.5Gyr ago. Nature, 485(7399):490-493, 2012. ISSN 00280836. doi: 10.1038/ nature11024. URL http://dx.doi.org/10.1038/nature11024.

[3] K. Chotalia, N. Cagney, C. Lithgow-Bertelloni, and J. Brodholt. The coupled effects of mantle mixing and a water-dependent viscosity on the surface ocean. Earth and Planetary Science Letters, 530:115881, jan 2020. ISSN 0012-821X. doi: 10.1016/J.EPSL.2019.115881. URL https://www. sciencedirect.com/science/article/pii/S0012821X19305734.

[4] U. R. Christensen. Thermal Evolution Models for the Earth. Journal of Geophysical Research, 90 (B4):2995-3007, 1985. ISSN 01480227. doi: 10.1029/JB090iB04p02995.

[5] K. C. Condie, R. C. Aster, and J. Van Hunen. A great thermal divergence in the mantle beginning 2.5 Ga: Geochemical constraints from greenstone basalts and komatiites. Geoscience Frontiers, 7(4):543-553, jul 2016. ISSN 16749871. doi: 10.1016/j.gsf.2016.01.006. URL https://www.sciencedirect.com/science/article/pii/S1674987116000311.

[6] J. W. Crowley and R. J. O'Connell. An analytic model of convection in a system with layered viscosity and plates. Geophysical Journal International, 188(1):61-78, 2012. ISSN 0956540X. doi: 10.1111/j.1365-246X.2011.05254.x.

[7] J. W. Crowley, M. Gérault, and R. J. O'Connell. On the relative influence of heat and water transport on planetary dynamics. Earth and Planetary Science Letters, 310(3-4):380-388, 2011. ISSN 0012821X. doi: 10.1016/j.epsl.2011.08.035. URL http://dx.doi.org/10.1016/j.eps1.2011.08. 035.

[8] G. F. Davies. Thermal histories of convective earth models and constraints on radiogenic heat production in the earth. Journal of Geophysical Research, 85(B5):2517-2530, 1980. ISSN 01480227. doi: 10.1029/JB085iB05p02517.

[9] J. Dong, R. A. Fischer, L. P. Stixrude, and C. R. Lithgow-Bertelloni. Constraining the Volume of Earth's Early Oceans With a Temperature-Dependent Mantle Water Storage Capacity Model. AGU Advances, 2(1), 2021. ISSN 2576-604X. doi: 10.1029/2020av000323. 
[10] J. Eguchi, J. Seales, and R. Dasgupta. Great Oxidation and Lomagundi events linked by deep cycling and enhanced degassing of carbon. Nature Geoscience, 13(1):71-76, jan 2020. ISSN 17520908. doi: 10.1038/s41561-019-0492-6. URL http://www.nature.com/articles/s41561-019-0492-6.

[11] J. Ganne and X. Feng. Geochemistry, Geophysics, Geosystems. Geochemistry Geophysics Geosystems, pages 1-26, 2017. ISSN 0012821X. doi: 10.1002/2016GC006679.Received. URL 10.1002/2016GC006787.

[12] G. Gerardi, N. M. Ribe, and P. J. Tackley. Plate bending, energetics of subduction and modeling of mantle convection: A boundary element approach. Earth and Planetary Science Letters, 515:47-57, jun 2019. ISSN 0012-821X. doi: 10.1016/J.EPSL.2019.03.010. URL https://www. sciencedirect . com/science/article/pii/S0012821X1930158X?via\%3Dihub.

[13] C. Herzberg, K. Condie, and J. Korenaga. Thermal history of the Earth and its petrological expression. Earth and Planetary Science Letters, 292(1-2):79-88, 2010. ISSN 0012821X. doi: 10.1016/j.epsl.2010.01.022. URL http://dx.doi.org/10.1016/j.epsl.2010.01.022.

[14] M. M. Hirschmann. Mantle solidus: Experimental constraints and the effects of peridotite composition. Geochemistry, Geophysics, Geosystems, 1(10), oct 2000. ISSN 15252027. doi: 10.1029/2000GC000070. URL https://agupubs.onlinelibrary.wiley.com/doi/full/10.1029/ 2000GC000070\%4010.1002/\%28ISSN\%291525-2027 . MANT1.

[15] G. Hirth and D. L. Kohlstedt. Water in the oceanic upper mantle: Implications for rheology, melt extraction and the evolution of the lithosphere. Earth and Planetary Science Letters, 144 (1-2):93-108, oct 1996. ISSN 0012821X. doi: 10.1016/0012-821x(96)00154-9. URL https://www . sciencedirect.com/science/article/pii/0012821X96001549.

[16] H. Iwamori. Transportation of H2O beneath the Japan arcs and its implications for global water circulation. Chemical Geology, 239(3-4):182-198, 2007. ISSN 00092541. doi: 10.1016/j.chemgeo. 2006.08.011.

[17] M. J. Jackson and H. N. Pollack. Mantle devolatilization and convection: implications for the thermal history of the Earth. Geophysical Research Letters, 14(7):737-740, 1987.

[18] A. Jambon. Chapter 12. EARTH DEGASSING AND LARGE-SCALE GEOCHEMICAL CYCLING OF VOLATILE ELEMENTS, pages 479-518. De Gruyter, 2018. doi: doi:10.1515/ 9781501509674-019. URL https://doi .org/10.1515/9781501509674-019.

[19] C. Jaupart, S. Labrosse, and J. C. Mareschal. Temperatures, Heat and Energy in the Mantle of the Earth, volume 7. Elsevier B.V., 2007. ISBN 9780444527486. doi: 10.1016/B978-044452748-6. 00114-0. URL http://dx.doi.org/10.1016/B978-0-444-53802-4.00126-3. 
[20] K. S. Karlsen, C. P. Conrad, and V. Magni. Deep Water Cycling and Sea Level Change Since the Breakup of Pangea. Geochemistry, Geophysics, Geosystems, page 2019GC008232, may 2019. ISSN 1525-2027. doi: 10.1029/2019GC008232. URL https://onlinelibrary.wiley.com/doi/abs/10. 1029/2019GC008232.

[21] R. F. Katz, M. Spiegelman, and C. H. Langmuir. A new parameterization of hydrous mantle melting. Geochemistry, Geophysics, Geosystems, 4(9):1-19, 2003. ISSN 15252027. doi: 10.1029/ 2002GC000433.

[22] B. Keller and B. Schoene. Plate tectonics and continental basaltic geochemistry throughout Earth history. Earth and Planetary Science Letters, 481:290-304, 2018. ISSN 0012821X. doi: 10.1016/j. epsl.2017.10.031.

[23] G. King and L. Zeng. The dangers of extreme counterfactuals. Political Analysis, 14(2):131-159, 2006. ISSN 10471987. doi: 10.1093/pan/mpj004.

[24] J. Korenaga. Energetics of mantle convection and the fate of fossil heat. Geophysical Research Letters, 30(8):47-63, 2003. ISSN 00948276. doi: 10.1029/2003GL016982.

[25] J. Korenaga. Can mantle convection be self-regulated? Science Advances, 2(8):e1601168-e1601168, aug 2016. ISSN 23752548. doi: 10.1126/sciadv.1601168. URL http://advances.sciencemag . org/ cgi/doi/10.1126/sciadv. 1601168 .

[26] C. T. A. Lee, L. Y. Yeung, N. R. McKenzie, Y. Yokoyama, K. Ozaki, and A. Lenardic. Two-step rise of atmospheric oxygen linked to the growth of continents. Nature Geoscience, 9(6):417-424, jun 2016. ISSN 17520908. doi: 10.1038/ngeo2707. URL http://www .nature.com/articles/ngeo2707.

[27] Z. X. A. Li, C. T. A. Lee, A. H. Peslier, A. Lenardic, and S. J. Mackwell. Water contents in mantle xenoliths from the Colorado Plateau and vicinity: Implications for the mantle rheology and hydration-induced thinning of continental lithosphere. Journal of Geophysical Research: Solid Earth, 113(9), 2008. ISSN 21699356. doi: 10.1029/2007JB005540.

[28] S. J. Mackwell, D. L. Kohlstedt, and M. S. Paterson. The role of water in the deformation of olivine single crystals. Journal of Geophysical Research, 90(B13):11319, nov 1985. ISSN 01480227. doi: 10.1029/jb090ib13p11319. URL http://doi.wiley.com/10.1029/JB090iB13p11319.

[29] P. J. McGovern and G. Schubert. Thermal evolution of the Earth: effects of volatile exchane between atmosphere and interior. Earth Planet. Sci. Lett, 96:27-37, 1989.

[30] D. Mckenzie and M. J. Bickle. The volume and composition of melt generated by extension of the lithosphere. Journal of Petrology, 29(3):625-679, 1988. ISSN 00223530. doi: 10.1093/petrology/29. 3.625 . 
[31] S. Mei and D. L. Kohlstedt. Influence of water on plastic deformation of olivine aggregates: 2. Dislocation creep regime. Journal of Geophysical Research: Solid Earth, 105(B9):21471-21481, sep 2000. ISSN 2169-9356. doi: 10.1029/2000jb900180. URL http://doi.wiley.com/10.1029/ 2000 JB900180.

[32] W. B. Moore and A. Lenardic. The efficiency of plate tectonics and nonequilibrium. Geophysical Research Letters, pages 9255-9260, 2015. doi: 10.1002/2015GL065621.1.

[33] R. Parai and S. Mukhopadhyay. Xenon isotopic constraints on the history of volatile recycling into the mantle. Nature, 560(7717):223-227, aug 2018. ISSN 0028-0836. doi: 10.1038/s41586-018-0388-4. URL http://www. nature.com/articles/s41586-018-0388-4.

[34] G. Schubert, D. Stevenson, and P. Cassen. Whole planet cooling and the radiogenic heat source contents of the earth and moon. Journal of Geophysical Research, 85(B5):2531-2538, 1980. ISSN 01480227. doi: 10.1029/JB085iB05p02531.

[35] G. Schubert, D. L. Turcotte, and P. Olson. Mantle convection in the earth and planets. Cambridge University Press, 2001. ISBN 9780511612879.

[36] J. Seales and A. Lenardic. Uncertainty Quantification in Planetary Thermal History Models: Implications for Hypotheses Discrimination and Habitability Modeling. The Astrophysical Journal, In Press, 2020.

[37] J. Seales and A. Lenardic. Deep Water Cycling and the Multi-Stage Cooling of the Earth. Geochemistry, Geophysics, Geosystems, 21(10):1-22, 2020. ISSN 15252027. doi: 10.1029/2020GC009106.

[38] J. Seales, A. Lenardic, and W. B. Moore. Assessing the Intrinsic Uncertainty and Structural Stability of Planetary Models: 1. Parameterized Thermal-Tectonic History Models. Journal of Geophysical Research: Planets, 124(8):2213-2232, aug 2019. ISSN 2169-9097. doi: 10.1029/2019je005918. URL https://onlinelibrary.wiley.com/doi/abs/10.1029/2019JE005918.

[39] D. J. Stevenson. Styles of mantle convection and their influence on planetary evolution. Comptes Rendus - Geoscience, 335(1):99-111, 2003. ISSN 16310713. doi: 10.1016/S1631-0713(03)00009-9.

[40] D. C. Tozer. The present thermal state of the terrestrial planets. Physics of the Earth and Planetary Interiors, 6(1-3):182-197, 1972. ISSN 00319201. doi: 10.1016/0031-9201(72)90052-0.

[41] H. J. Van Avendonk, J. K. Davis, J. L. Harding, and L. A. Lawver. Decrease in oceanic crustal thickness since the breakup of Pangaea. Nature Geoscience, 10(1):58-61, 2017. ISSN 17520908. doi: $10.1038 /$ ngeo2849. 